$\begin{array}{ll} & \text { Preprints are preliminary reports that have not undergone peer review. } \\ \text { Research Square } & \text { They should not be considered conclusive, used to inform clinical practice, } \\ \text { or referenced by the media as validated information. }\end{array}$

\title{
Chest Computed Tomography for SARS-CoV-2 Screening before Bariatric and Metabolic Surgery during the COVID-19 Pandemic
}

\section{Elias Ortiz-Gomez}

Department of Bariatric Surgery, Baja Hospital \& Medical Center, Tijuana, Mexico.

Manuel Alberto Guerrero-Gutierrez

Department of Critical Care, Instituto Nacional de Cancerologia, Mexico City, Mexico. https://orcid.org/0000-0002-0645-1836

Almino Ramos Cardoso

Department of Gastro-Obeso-Center Institute of Metabolic Optimization, Sao Pablo, Brazil. https://orcid.org/0000-0001-6805-1750

Patricia Ruiz-Cota ( $\sim$ ruizp@uabc.edu.mx)

Department of Bariatric Surgery, Baja Hospital \& Medical Center, Tijuana, Mexico. https://orcid.org/0000-0002-3115-2942

Diego Escarraman-Martinez

Departamento de Anestesiología, Hospital de Especialidades, CMN La Raza IMSS, Mexico City, Mexico. https://orcid.org/0000-0003-3190-0258

Cynthia Rios

Department of Bariatric Surgery, Baja Hospital \& Medical Center, Tijuana, Mexico.

Maria Hernandez-Barrozo

Department of Bariatric Surgery, Baja Hospital \& Medical Center, Tijuana, Mexico.

Orlando Ruben Perez Nieto

Department of Critical Care, Hospital General San Juan del Río, San Juan del Río, Queretaro, Mexico.

\section{Research Article}

Keywords: Bariatric and metabolic surgery, Computed tomography, CT chest scan, Safe surgery, SARS-CoV-2, COVID-19

Posted Date: October 30th, 2020

DOI: https://doi.org/10.21203/rs.3.rs-99050/v1

License: @ (i) This work is licensed under a Creative Commons Attribution 4.0 International License. Read Full License 


\section{Abstract}

Introduction: Given that obesity is a risk factor for adverse outcomes in COVID-19, bariatric and metabolic surgery (BMS) has become increasingly critical. Computed tomography (chest CT scan) may be a valuable preoperative screening method for BMS candidates during this COVID-19 pandemic.

Methods: A prospective, single-center study was conducted in June 2020. Two study cohorts were evaluated: a surgical team group and a BMS patient group. Screening was performed with RT-PCR and a CT scan. The COVID-19 Reporting and Data System (CO-RADS) assessment was used as mandatory COVID-19 screening. Patients classified as category 1 and 2 were considered safe to undergo BMS; category $\geq 3$ surgery was canceled. The BMS patient group was monitored for 28 days after surgery for SARS-CoV-2 infection. Additionally, postoperative complications, adverse effects, and deaths were tracked.

Results: The study included 240 participants, comprising the surgical team group $(n=6)$ and the BMS patient group $(n=234)$. In total, 213 were female (88.8\%), the median age was 40 years, and the median weight and BMI were $111.1 \mathrm{~kg}$ and $40.23 \mathrm{~kg} / \mathrm{m}^{2}$ respectively. Only CO-RADS category 1 was reported in the surgical team group, while in the BMS patient group, category 1 was reported in 231 participants (98.7\%). In the BMS patient group, during the follow-up period, only two participants $(0.83 \%)$ tested positive for COVID-19 (RT-PCR). No deaths were reported.

Conclusion: Chest CT scans are useful for detecting SARS-CoV-2 in patients undergoing BMS, therefore enable healthcare and surgical teams to perform surgeries safely during this COVID-19 pandemic.

\section{Introduction}

Elective bariatric and metabolic surgery (BMS) has slowed down worldwide. While it has not ground to a complete halt, the number of surgeries has decreased drastically during the COVID-19 pandemic [1]. The inability to maintain an active lifestyle and adhere to lifestyle interventions due to quarantining and lockdowns has provided the perfect conditions for an increase in the global burden of obesity. As it is widely known that one of the most important risk factors for adverse outcomes in COVID-19 is obesity, BMS has become increasingly important and constitutes a priority at this point [2]. From a patient-centered and public health standpoint, it is critical to resume BMS. There is clear evidence that BMS improves survival and significantly improves severe critical obesityrelated comorbidities (including hypertension, type 2 diabetes mellitus, and cerebrovascular disease), which are also known to be severity risk factors for COVID-19 infection [2-4].

The evolution and impact of the COVID-19 pandemic varies greatly from country to country [5]. SARS-CoV-2 will probably circulate in the population for a long time, and the return to normal services, including surgery, will be gradual. Factors that need to be considered to resume BMS include the local prevalence of COVID-19 and the availability of screening tests, health care workers, and resources such as hospital beds, ventilators, and personal protective equipment [2]. However, a delay of months or years in life-saving survival treatment of obesity and its repercussions is not in the best interest of our patients, and the risk and degree of harm from delaying BMS would vary based on each patient's condition. An early resumption of elective BMS could improve metabolic and immunological conditions in these patients, putting them at lower risk of developing serious complications from COVID-19 and probably reducing that risk to the same level as the general population [2,5,6]. While we stress the importance of resuming BMS, we acknowledge it is important to gain a thorough understanding of the limitations of resources in every country, city, and hospital in order to wisely select screening and diagnostic tests suitable for candidates for BMS.

The International Federation for the Surgery of Obesity and Metabolic Disorders (IFSO) has recommended standards for patient care during the pandemic, such as vital signs monitoring, computed tomography of the chest (chest CT), and reverse transcription polymerase chain reaction (RT-PCR) tests for SARSCoV-2 [7]. The chest CT scan is considered a cornerstone in the evaluation of this disease [8-12]. Given the risk of severe complications from COVID-19 in patients with obesity, mandatory preoperative screening should be recommended for all patients considering BMS.

In addition to the need to preserve resources for potential COVID-19 patient care and minimize unnecessary COVID-19 exposure, Lei S et al., Aminian A et al., together with the COVIDSurg Collaborative, demonstrated an increased risk of mortality (of $20.6 \%, 75 \%$, and $23.8 \%$, respectively) in patients undergoing general surgery with acute COVID-19 and in patients undergoing general surgery with COVID-19 complicated by severe pulmonary infection [8,13,14]. Therefore, to enable a safe bariatric surgery program, an adequate diagnostic method to identify patients in the acute phase of COVID-19 proves critical [15]. The most commonly used diagnostic test worldwide for SARS-CoV-2 infection is RT-PCR; however, this method has failed to recognize more than $29 \%$ of asymptomatic patients [16]. Additionally, the shortage of RT-PCR kits in many countries raises serious concerns for screening patients before surgery [17,18]. However, if the patients are not accurately screened, there is a risk of the health personnel involved contracting SARS-CoV-2 during the preoperative evaluations and surgical procedure. Orotracheal intubation and the surgical use of an electrocautery, ultrasonic device, and laser are considered risk factors for infecting the surgical team by pneumoperitoneum aerosolization [19-22].

Based on the elevated risk of mortality in patients with the pulmonary form of the COVID-19 infection, the routine use of a chest CT scan prior to elective BMS appears to be a good, acceptable, and valuable SARS-CoV-2 screening tool $[15,23]$.

Page $2 / 11$ 
In a recent meta-analysis of chest CT images from 1,115 COVID-19 patients, Wan et al. [11] described a pattern of characteristic imaging findings, namely ground-glass opacities and pleural thickening (69\%), peripheral distribution (70\%), consolidation (70\%), air bronchograms (46\%), right lower lobe involvement (70\%), involvement of more than three lobes (15\%), and an irregular paving pattern (15\%).

Moreover, other studies have demonstrated that chest CT can be a valuable diagnostic method for patients with suspected COVID-19 because it has a sensitivity of up to $97 \%[10,11]$. A complete understanding of the chest CT patterns and clinical characteristics of patients with COVID-19 can be beneficial for effectively diagnosing and treating patients with COVID-19 when evaluating patients for BMS. However, the classification by means of so-called categories has a great impact on screening [24,25], which, due to the lack of diagnostic tests, is a fundamental pillar in the decision-making process. Therefore, we assessed whether it is possible to safely perform elective BMS during the COVID-19 pandemic without exposing the patient or medical personnel, in an attempt to make a safe return to surgeries.

\section{Methods}

Prior to conducting this study, the researchers took into account the recommendations issued by different professional groups such as the IFSO and ASMBS $[5-7,26]$. These groups considered BMS essential, and therefore the safety goals were met and adequate triage was performed to identify patients with potential SARS-CoV-2 infection.

\section{Participant Selection}

This study was evaluated and approved by the local ethics and research committee of the hospital. Each patient gave written informed consent for their data to be used for research and publication.

This research included two study groups: the team of health care professionals (surgical team group) and the patients scheduled for elective BMS (BMS patient group). The inclusion criteria for the BMS patient group included U.S. national patients scheduled for elective BMS at the Baja Hospital \& Medical Center in June 2020. Elimination criteria included patients under 18 years old, BMI $<30 \mathrm{~kg} / \mathrm{m}^{2}$, and chronic diseases that contraindicate the surgical procedure (e.g., lung cancer). Exclusion criteria included patients with a diagnosis of COVID-19, patients with symptoms related to COVID-19 (e.g., fever), and patients with an infectious disease for which BMS was contraindicated or not recommended at that specific time (e.g., community-acquired pneumonia). The group selection schema is shown in Figure 1. The surgical team group included the surgeon, the surgeon's surgical assistant, the anesthesiologist, and the surgical nurse and circulating nurses, and the same personnel were involved in all surgical procedures.

Preoperative Assessment and COVID-19 Screening

Both groups were screened to confirm the absence of SARS-CoV-2 infection prior to surgery; in the surgical team group, COVID-19 status was determined before the study began (May 28th), using chest CT scans and RT-PCR as diagnostic tools. Due to the limitations of RT-PCR and a shortage of RT-PCR kits (which are mainly destined for public hospitals and health care workers), as well as handling and processing difficulties (especially in that period of time in our locality and country), it was not possible to use the RT-PCR as a diagnostic tool in the BMS patient group. However, on the basis of previous good diagnostic performance in asymptomatic individuals [23], chest CT scans were employed as mandatory COVID-19 screening and categorized according to the COVID-19 Reporting and Data System (CO-RADS) (Table 1). Patients classified as category 1 (very low suspicion of COVID-19) and category 2 (low suspicion of COVID19) were considered safe to perform BMS, and for patients classified as category $\geq 3$ (indeterminate to very high suspicion of COVID-19), BMS was canceled. The chest CT scans were performed preoperatively, one day before surgery or in some cases on the day of surgery (depending on the day of arrival and the scheduled date of surgery).

For the BMS patient group, a preoperative selection algorithm was developed in order to follow a standardized protocol (Figure 2) from the patients' arrival to their admission into the hospital. Additionally, in the BMS patient group, a complete preoperative assessment was performed, including a verification of the COVID-19 self-assessment (Appendix 1) and COVID-19 questionnaire (Appendix 2), a preoperative evaluation by the Internal Medicine Department that included the patient's medical history, a physical examination, and assessment of clinical characteristics (weight, height, BMl, and comorbidities). 
Four standardized types of laparoscopic BMS were performed: sleeve gastrectomy (SG), Roux-en-Y gastric bypass (RYGB), one anastomosis gastric bypass (OAGB), and bariatric revisional surgery. Surgery selection was based on the individual needs of each patient. All surgeries were conducted under general anesthesia. The surgical team group wore full protection (surgical equipment, N95 or P100 masks, and face shield, depending on personal preference) during all the procedures.

Follow-Up and Outcome Assessment

In the surgical team group, COVID-19 status was determined by RT-PCR and chest CT scan after the surgical procedures for June were completed (July 3). The BMS patient group was followed up for 28 days after surgery to monitor for SARS-CoV-2 infection (the primary outcome) and the development of postoperative complications, adverse effects, and deaths (secondary outcomes). The postoperative follow-up of this group was performed via phone calls by a certified bariatric nurse from the American Society for Metabolic and Bariatric Surgery (ASMBS) on the 14th and 28th postoperative day. The follow-up phone calls consisted in directed questioning to determine the development or presentation of symptoms or signs of COVID-19. If patients reported positive symptoms or signs of COVID-19 in the follow-up period, they were asked to take a RT-PCR test to confirm the presence of the disease. Postoperative complications, adverse effects, and deaths were also assessed by phone calls on the 14th and 28th day after surgery. These calls consisted in a debriefing focused on the most common immediate complications of BMS (e.g., wound problems, pulmonary embolism, leaks, stenosis, internal hernias, port-site hernias, etc.).

Statistical Analysis

For the statistical analysis of this study, firstly we analyzed the clinical characteristics, including age, sex, weight, height, BMI, and comorbidities including hypertension (HTN), type 2 diabetes mellitus (DM2), obstructive sleep apnea syndrome (OSA), hypothyroidism, dyslipidemia, psychosocial disorders, and others (musculoskeletal disorders, migraine, insomnia, and dermatologic disorders) for the entire sample of participants. Then the sample was divided into groups-the surgical team group and the BMS patient group-for which the clinical characteristics and comorbidities were examined. Subsequently, the participants from the BMS patient group were stratified into subgroups based on the surgical technique performed (SG, RYGB, OAGB, bariatric revisional surgery), and finally, COVID-19 and postoperative complications during the follow-up period (phone calls on the 14th and 28th day after surgery) were reported. Descriptive statistics were used to evaluate the primary and secondary outcomes.

To ensure the quantitative variables were correctly reported, the data distribution was first verified with the Kolmogorov-Smirnov test; variables with normal distribution are presented as the mean and standard deviation, and variables that were not normally distributed are presented as the median and interquartile range (IQR). Nominal and categorical variables are presented as proportions; absolute frequency and percentages were used. SPSS version 25 software was used for statistical analysis.

\section{Results}

Initially, a sample of 250 participants was selected for the study: six participants for the surgical team group and 244 participants for the BMS patient group. Five participants from the BMS patient group were eliminated: three participants under 18 years old, one with a BMI $<30 \mathrm{~kg} / \mathrm{m}^{2}$, and another who presented diagnosis of lung cancer. This left 239 participants for the BMS patient group; five participants were then eliminated due to exclusion criteria: 3 participants presented CO-RADS category $\geq 3$, one presented fever before surgery, and another was diagnosed with community-acquired pneumonia (not COVID-19) based on the chest CT scan results. A total of 240 participants were included in the final study: six participants for the surgical team group and 234 participants for the BMS patient group. The group selection schema is shown in Figure 1.

In total, 213 participants were female, representing $88.8 \%$ of the sample, while 27 were male, equivalent to $11.2 \%$; the median age was 40 years, with an interquartile range (IQR) of 16 years; the median weight was $111.1 \mathrm{~kg}$, with an IQR of $33.4 \mathrm{~kg}$; the mean height was $1.66 \mathrm{~m}$, with an IQR of $0.1 \mathrm{~m}$; and the mean BMI was $40.23 \mathrm{~kg} / \mathrm{m}^{2}$, with an IQR of 11.6 . In terms of the CO-RADS classification, only category 1 was reported in the surgical team group (100\%). In the BMS patient group, categories 1 (very low suspicion of COVID-19) and 2 (low suspicion of COVID-19) were reported, with an absolute frequency of 231 patients classified as category 1 (98.7\%), while only 3 patients were classified as category 2 (1.3\%). A summary of the characteristics of each group is shown in Table 2. 
The BMS patient group was also stratified according to the type of surgical procedure performed: the most common type of procedure was SG, representing $81.6 \%$ of the total $(n=191)$, while the least common was the RYGB procedure, with only $2.1 \%(n=5)$. Heavier patients were observed in the RYGB group, with a median weight of $134.9 \mathrm{~kg}$ and an IQR of $46.4 \mathrm{~kg}$. Comorbidities were observed as follows: HTN 17.6\% $(n=60)$, DM2 7.3\% ( $n=25), 0 S A 5.5 \%(n=19)$, hypothyroidism 7.3\% ( $n=25)$, dyslipidemia $5 \%(n=17)$, psychosocial disorders $27.6 \%(n=94)$, and others present in $29.4 \%(n=100)$. A summary of the clinical variables and comorbidities of patients reclassified by surgical technique is shown in Table 3.

For the primary outcome, none of the participants in the surgical team group tested positive for COVID-19 (RT-PCR and chest CT scan) after performing the surgical procedures. On the other hand, in the BMS patient group, two patients reported symptoms and signs associated with COVID-19 during the follow-up period. RT-PCR was requested and yielded positive results for COVID-19 in these two patients, one at 21 days and the other at 24 days after surgery, indicating an incidence rate of only $0.83 \%$ during the follow-up period.

Regarding secondary outcomes, five patients (2.1\%) reported postoperative complications. Four patients (1.7\%) experienced complications during the hospital stay: three patients had postoperative intraperitoneal bleeding (1.2\%), and one patient required reoperation due to gastric sleeve obstruction ( $0.42 \%)$. One other patient $(0.42 \%)$ presented with a late complication 22 days after surgery (gastric sleeve stenosis). During the 28-day follow-up period, no deaths were reported.

\section{Discussion}

In our study, we identified three asymptomatic patients with indeterminate to high suspicion of COVID-19 according to the CO-RADS assessment; therefore, these patients did not undergo BMS. During the follow-up period, only two participants (0.83\%) reported compatible symptoms and signs of COVID-19. RT-PCR was requested, and they both tested positive for COVID-19 (21 and 24 days after surgery). On the other hand, the surgical team did not present any positive RTPCR results on either of the two sampling dates (May 28 and July 3). Therefore, we can report that BMS patients and surgical team members screened as CORADS category 1 or 2 on the chest CT scan had a low incidence of COVID-19 after potential exposure (surgery), indicating that the chest CT scan can determine which patients it is safe to perform BMS on.

Postoperative complications were reported in five patients (2.1\%). Four (1.7\%) had complications reported during their hospital stay (three of whom [1.2\%] had postoperative intraperitoneal bleeding), and one patient (0.42\%) presented with a complication after hospital discharge that required surgical reintervention due to gastric sleeve stenosis, with symptoms similar to those described by Taube et al. [27]. During the 28-day follow-up, there were no deaths reported.

To reduce unnecessary risk of COVID-19 transmission among and between patients and health care personnel in relation to elective procedures, screening for SARS-CoV-2 infection with a highly sensitive diagnostic upon admission should be mandatory. RT-PCR is considered the standard test for COVID-19 diagnosis; however, this method has notable limitations, such as a shortage of kits, inadequate lab capability, and problems associated with sample collection and transportation, which can culminate in false negative results in the early stages of the disease $[17,18]$. Thus, given previous good performance in detecting asymptomatic individuals [21], chest CT scans categorized according to the CO-RADS assessment were used in this study as a cornerstone of preoperative screening. A proposed diagram for preoperative COVID-19 screening in individuals undergoing BMS is presented in Figure 2.

In a study conducted by De Smet et al. [23], chest CT scans using CO-RADS scoring represented a good diagnostic tool for both symptomatic and asymptomatic patients. Likewise, Shao et al. [15] carried out a systematic review and concluded that CT should be considered for the detection of COVID-19; additional studies have proposed that chest CT should be used even in asymptomatic COVID-19 patients [28] and as a preoperative assessment for all patients [29].

Interestingly, all the studies published so far have reported good, adequate, and safe performance of chest CT scans as a screening tool for symptomatic and asymptomatic COVID-19 individuals. Therefore, we propose that chest CT scans can be used as a COVID-19 diagnostic test to screen patients before they undergo BMS. Prospective randomized controlled studies are required to corroborate these results.

Our study has the following limitations: 1) COVID-19 prevention efforts through social networks (patient education before arrival about symptoms and signs of COVID-19) meant that symptomatic patients chose not to make the trip for surgery, so fewer cases of COVID-19 were detected on the chest CT scans; 2) RTPCR was not directly compared with chest CT scans; and 3) the single-center study design made it impossible to observe the reproducibility of this screening approach.

\section{Conclusion}

Preoperative chest CT scans for detecting SARS-CoV-2 infection were shown to be an excellent alternative for screening during this COVID-19 pandemic. Taking into consideration the low sensitivity of RT-PCR tests in asymptomatic individuals, as well as the lack of kits and handling and processing difficulties in many countries, chest CT scans offer a highly valuable tool for preoperative screening. Further prospective randomized controlled studies are warranted to corroborate our results.

\section{Declarations}

\section{Ethical considerations}


This research project was carried out under the ethical precepts indicated in the General Health Law, Regulations on Research for Health, Official Mexican Standard 012 (NOM-012). This research did not pose any additional risk to the study participants, and the data were obtained from the clinical files of the patients undergoing elective surgery during the month of June 2020. The researchers in the group were committed to maintaining the confidentiality of the data in the files and to safeguarding the identities of patients for academic and scientific purposes. Within the scope of this project, a screening protocol was implemented to assess the possibility of bariatric surgery during the COVID-19 pandemic.

\section{Authors' contributions}

EOG \& MAGG were responsible for the conceptualization of the study and the revision and approval of this manuscript. DEM performed the statistical analysis, and PRC \& ORPN drafted the manuscript and were responsible for its accuracy. ARC critically reviewed the paper. All the authors read and approved the final version of the manuscript.

\section{Funding}

We have not received any type of support.

\section{Conflict of interest}

We declare that we have no conflicts of interest.

\section{Availability of data and materials}

The data are available in the medical records system of the Baja Hospital \& Medical Center.

\section{Ethics approval and consent to participate}

This study was approved by the ethics committee of Baja Hospital \& Medical Center.

\section{Consent for publication}

Each patient gave written informed consent for their data to be used for research and publication.

\section{Competing interests}

The authors declare that they have no competing interests.

\section{References}

1. Hussain A, Mahawar K, El-Hasani S. The Impact of COVID-19 Pandemic on Obesity and Bariatric Surgery. Obes Surg. 2020 Aug;30(8):3222-3223.

2. Cummings DE, Rubino F, Schauer PR, Cohen RV. Bariatric and metabolic surgery during and after the COVID-19 pandemic-Authors' reply. Lancet Diabetes Endocrinol. 2020 Sep;8(9):743-744.

3. Wolff D, Nee S, Hickey NS, Marschollek M. Risk factors for Covid-19 severity and fatality: a structured literature review. Infection. 2020 Aug 28:1-14.

4. Popkin BM, Du S, Green WD, Beck MA, Algaith T, Herbst CH, et al. Individuals with obesity and COVID-19: A global perspective on the epidemiology and biological relationships. Obes Rev. 2020 Aug 26:10.1111/obr.13128.

5. Behrens E, Poggi L, Aparicio S, Martínez Duartez P, Rodríguez N, Zundel N, et al. COVID-19: IFSO LAC Recommendations for the Resumption of Elective Bariatric Surgery. Obes Surg. 2020 Nov;30(11):4519-4528.

6. Marinari GM, Anselmino M, Tascini C, Bernante P, Foletto M, Gentileschi P, et al. Bariatric and metabolic surgery during COVID-19 outbreak phase 2 in Italy: why, when and how to restart. Surg Obes Relat Dis. 2020 Jun 24;16(10):1614-8.

7. Dong Z, Zhang P, Zhu J, Bai B, Parmar C, Chen W, et al. Recommendations to Manage Patients for Bariatric Surgery in the COVID-19 Pandemic: Experience from China. Obes Surg. 2020 June; 30:4623-4626.

8. Aminian A, Kermansaravi M, Azizi S, Alibeigi P, Safamanesh S, Mousavimaleki A, et al. Bariatric Surgical Practice During the Initial Phase of CoVID-19 Outbreak. Obes Surg. 2020 Sep;30(9):3624-3627.

9. Nahshon C, Bitterman A, Haddad R, Hazzan D, Lavie O. Hazardous Postoperative Outcomes of Unexpected COVID-19 Infected Patients: A Call for Global Consideration of Sampling all Asymptomatic Patients Before Surgical Treatment. World J Surg. 2020 Aug;44(8):2477-2481.

10. Lomoro P, Verde F, Zerboni F, Simonetti I, Borghi C, Fachinetti C, et al. COVID-19 pneumonia manifestations at the admission on chest ultrasound, radiographs, and CT: single-center study and comprehensive radiologic literature review. Eur J Radiol Open. 2020;7:100231.

11. Wan S, Li M, Ye Z, Yang C, Cai Q, Duan S, et al. CT Manifestations and Clinical Characteristics of 1115 Patients with Coronavirus Disease 2019 (COVID19): A Systematic Review and Meta-analysis. Acad Radiol. 2020 Jul;27(7):910-921.

12. Ai T, Yang Z, Hou H, Zhan C, Chen C, Lv W, et al. Correlation of Chest CT and RT-PCR Testing for Coronavirus Disease 2019 (COVID-19) in China: A Report of 1014 Cases. Radiology. 2020 Aug;296(2):E32-E40.

13. Lei S, Jiang F, Su W, Chen C, Chen J, Mei W, et al. Clinical characteristics and outcomes of patients undergoing surgeries during the incubation period of COVID-19 infection. EClinicalMedicine. 2020 Apr 5;21:100331. 
14. COVIDSurg Collaborative. Mortality and pulmonary complications in patients undergoing surgery with perioperative SARS-CoV-2 infection: an international cohort study. Lancet. 2020 Jul 4;396(10243):27-38.

15. Shao JM, Ayuso SA, Deerenberg EB, Elhage SA, Augenstein VA, Heniford BT. A systematic review of CT chest in COVID-19 diagnosis and its potential application in a surgical setting. Colorectal Dis. 2020 Jul 9:10.1111/codi.15252.

16. Arevalo-Rodriguez I, Buitrago-Garcia D, Simancas-Racines D, Zambrano-Aching P, Del Campo R, Ciapponi A, et al. False-negative results of initial RT-PCR assays for COVID-19: a systematic review. medRxiv 2020.

17. Rodriguez-Morales AJ, Gallego V, Escalera-Antezana JP, Méndez CA, Zambrano LI, Franco-Paredes C, et al. COVID-19 in Latin America: The implications of the first confirmed case in Brazil. Travel Med Infect Dis. 2020 May-Jun;35:101613.

18. Hadaya J, Schumm M, Livingston EH. Testing Individuals for Coronavirus Disease 2019 (COVID-19).JAMA.2020;323(19):1981.

19. Bra Brat GA, Hersey S, Chhabra K, Gupta A, Scott J. Protecting Surgical Teams During the COVID-19 Outbreak: A Narrative Review and Clinical Considerations. Ann Surg. 2020 Apr 17:10.1097/SLA.0000000000003926.

20. Kwak HD, Kim SH, Seo YS, Song KJ. Detecting hepatitis B virus in surgical smoke emitted during laparoscopic surgery. Occup Environ Med. 2016 Dec;73(12):857-863.

21. Stephenson DJ, Allcott DA, Koch M. The presence of P22 bacteriophage in electrocautery aerosols. In: Proceedings of the National Occupational Research Agenda Symposium, Salt Lake City, UT Available from:

https://www.researchgate.net/profile/Dale_Stephenson/publication/242419093_THE_PRESENCE_OF_P22_BACTERIOPHAGE_IN_ELECTROCAUTERY_AER d52a09d15f05f3000000.pdf. 2004.

22. Francis N, Dort J, Cho E, Feldman L, Keller D, Lim R, et al. SAGES and EAES recommendations for minimally invasive surgery during COVID-19 pandemic. Surg Endosc. 2020;34(6):2327-2331.

23. De Smet K, De Smet D, Ryckaert T, Laridon E, Heremans B, Vandenbulcke R, et al. Diagnostic Performance of Chest CT for SARS-CoV-2 Infection in Individuals with or without COVID-19 Symptoms. Radiology. 2020 Aug 10:202708.

24. Prokop M, Everdingen W, Rees VT, Quarles van Ufford H, Stöger L, Beenen L, et al. CO-RADS: A Categorical CT Assessment Scheme for Patients Suspected of Having COVID-19-Definition and Evaluation. Radiology 2020; 296: E97-E104.

25. Bai H, Hsieh B, Xiong Z, Halsey K, Whae Choi J, Linh Tran TM, et al. Performance of Radiologists in Differentiating COVID-19 from Non-COVID-19 Viral Pneumonia at Chest CT. Radiology 2020; 296: E46-E54.

26. Da Silva LE, Cohen RV, De-Andrade JC, Szegö T, Santo MA, Ramos AC. The recommendations of the Brazilian College of Surgeons and the Brazilian Bariatric and Metabolic Surgery Societies on the return of bariatric and metabolic operations in geographic regions of the country where the procedures have been allowed by local policies, in the period of COVID-19 pandemic. Rev Col Bras Cir 47: e20202640.

27. Taube C, Mansour S, Hakky S. A Unique Presentation of COVID-19 in a Patient Post Sleeve Gastrectomy. Obes Surg. 2020 May; 9: 1-2.

28. Meng H, Xiong R, He R, Lin W, Hao B, Zhang L, et al. CT imaging and clinical course of asymptomatic cases with COVID-19 pneumonia at admission in Wuhan, China. Journal of Infection 81 (2020) e33-e39.

29. Callaway M, Harden S, Ramsden W, Beavon M, Drinkwater K, Vanburen T, et al. A national UK audit for diagnostic accuracy of preoperative CT chest in emergency and elective surgery during COVID-19 pandemic. Clinical Radiology 75 (2020) 705e708.

\section{Tables}

Table 1. Chest CT assessment using the CO-RADS categorical assessment scheme to evaluate suspicion of COVID-19

\begin{tabular}{|lll|}
\hline Category & Level of COVID-19 suspicion & Chest CT findings \\
\hline CO-RADS 1 & Very low & Normal or noninfectious abnormalities \\
\hline CO-RADS 2 & Low & Abnormalities consistent with infections other than COVID-19 \\
\hline CO-RADS 3 & Indeterminate & Unclear whether COVID-19 is present \\
\hline CO-RADS 4 & High & Abnormalities suspicious for COVID-19 \\
CO-RADS 5 & Very high & Typical COVID-19 \\
\hline CO-RADS 6 & Proven & RT-PCR + for SARS-CoV-2 \\
\hline
\end{tabular}

CO-RADS = COVID-19 Reporting and Data System; COVID-19 = coronavirus disease 2019; RT-PCR = reverse transcription polymerase chain reaction; SARSCoV-2 = severe acute respiratory syndrome coronavirus 2. Modified from Prokop et al., 2020 [23].

Table 2. Clinical and comorbidities characteristics of the participants in BMS patient group $(n=234)$ and surgical team group $(n=6)$. 


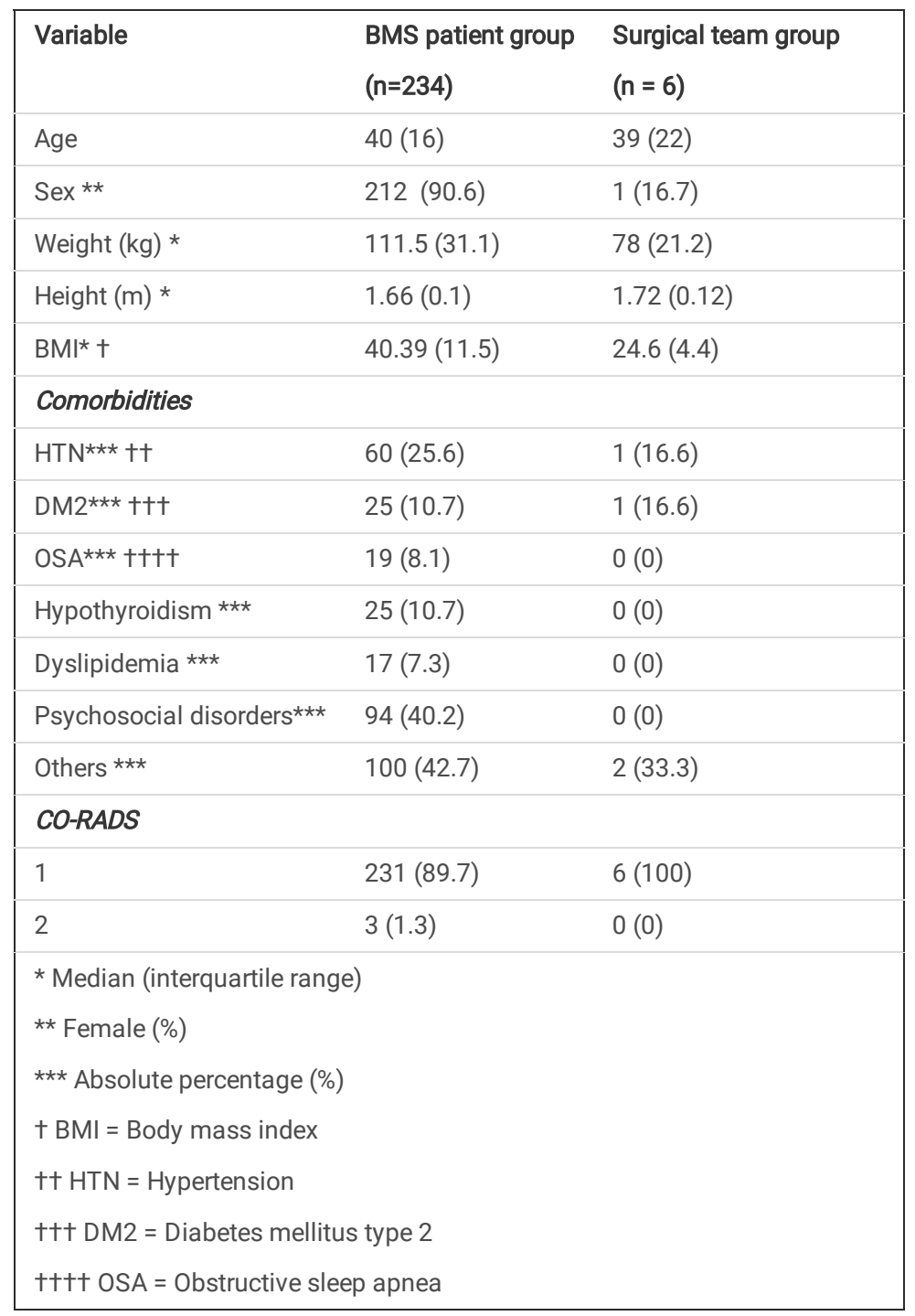

Table 3. Clinical and comorbidities characteristics of the participants in the BMS patient group ( $n=234)$ stratified by type of surgery. 


\begin{tabular}{|c|c|c|c|c|c|}
\hline Variable & $\begin{array}{l}\text { Sleeve } \\
\text { gastrectomy } \\
(n=191)\end{array}$ & $\begin{array}{l}\text { Roux-en-Y gastric } \\
\text { bypass } \\
(n=7)\end{array}$ & $\begin{array}{l}\text { One anastomosis gastric } \\
\text { bypass } \\
(n=5)\end{array}$ & $\begin{array}{l}\text { Bariatric revision } \\
\text { surgery } \\
(n=31)\end{array}$ & Total \\
\hline Age & $38(16)$ & $40(13)$ & 44 (19) & $44(13)$ & $40(16)$ \\
\hline Sex ** & $173(90.6)$ & $6(85.7)$ & $4(80)$ & $29(93.5)$ & $212(90.6)$ \\
\hline Weight $(\mathrm{kg})$ * & $111.5(32.3)$ & $111.5(30.4)$ & $134.9(46.4)$ & $107(47.8)$ & $\begin{array}{l}111.5 \\
(31.1)\end{array}$ \\
\hline Height $(m)$ * & $1.66(0.1)$ & $1.62(0.16)$ & $1.71(0.23)$ & $1.65(0.12)$ & $1.66(0.1)$ \\
\hline $\mathrm{BMI}^{*}+$ & $40.3(10)$ & $41.5(12.9)$ & $48.4(10)$ & $38.2(12.2)$ & $\begin{array}{l}40.39 \\
(11.5)\end{array}$ \\
\hline \multicolumn{6}{|l|}{ Comorbidities } \\
\hline HTN $N^{\star \star \star}+†$ & $54(28.3)$ & $0(0)$ & $2(40)$ & $4(12.9)$ & $60(17.6)$ \\
\hline 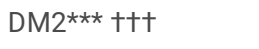 & $20(10.5)$ & $2(28.6)$ & $2(40)$ & $1(3.2)$ & $25(7.3)$ \\
\hline 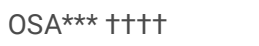 & $16(8.4)$ & $1(14.3)$ & $1(20)$ & $1(3.2)$ & $19(5.5)$ \\
\hline Hypothyroidism *** & $16(8.4)$ & $1(14.3)$ & $3(60)$ & $5(16.1)$ & $25(7.3)$ \\
\hline 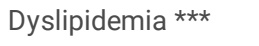 & $11(5.8)$ & $0(0)$ & $2(40)$ & $4(12.9)$ & $17(5)$ \\
\hline $\begin{array}{l}\text { Psychosocial } \\
\text { disorders }\end{array}$ & 75 (39.3) & $1(14.3)$ & $3(60)$ & $15(48.4)$ & $94(27.6)$ \\
\hline Others *** & $74(38.7)$ & $4(57.1)$ & $3(60)$ & $19(61.3)$ & $100(29.4)$ \\
\hline 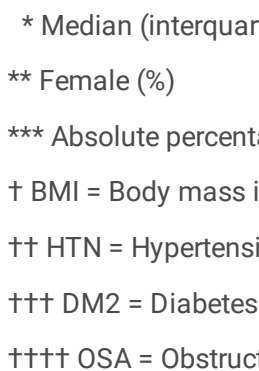 & s type 2 & & & & \\
\hline
\end{tabular}

\section{Figures}




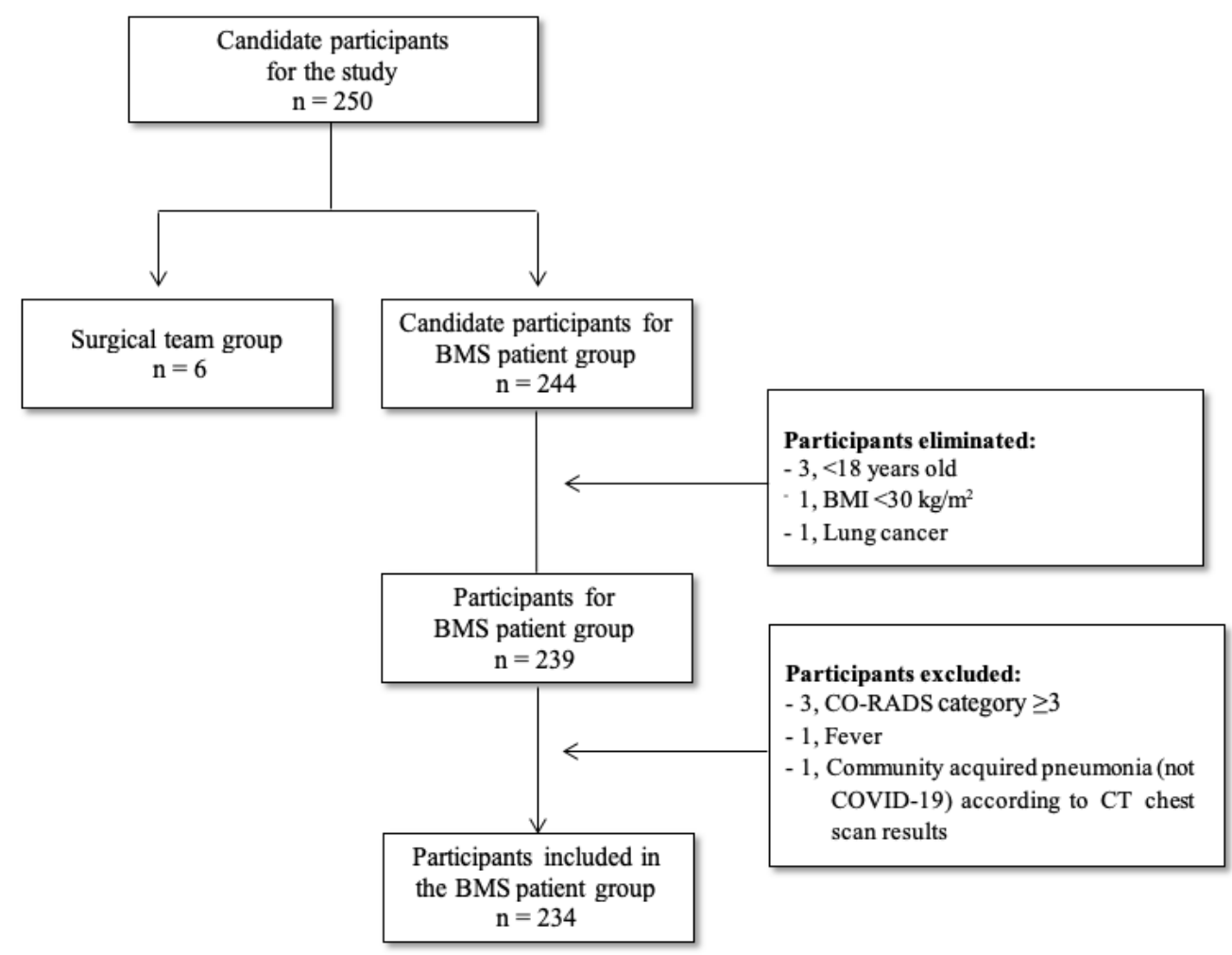

Figure 1

Participants selection schema for BMS patient group. BMS = Bariatric and metabolic surgery; CT chest $=$ Computed tomography of the chest; $\operatorname{COVID}-19=$ coronavirus disease 2019; BMI = body mass index. 


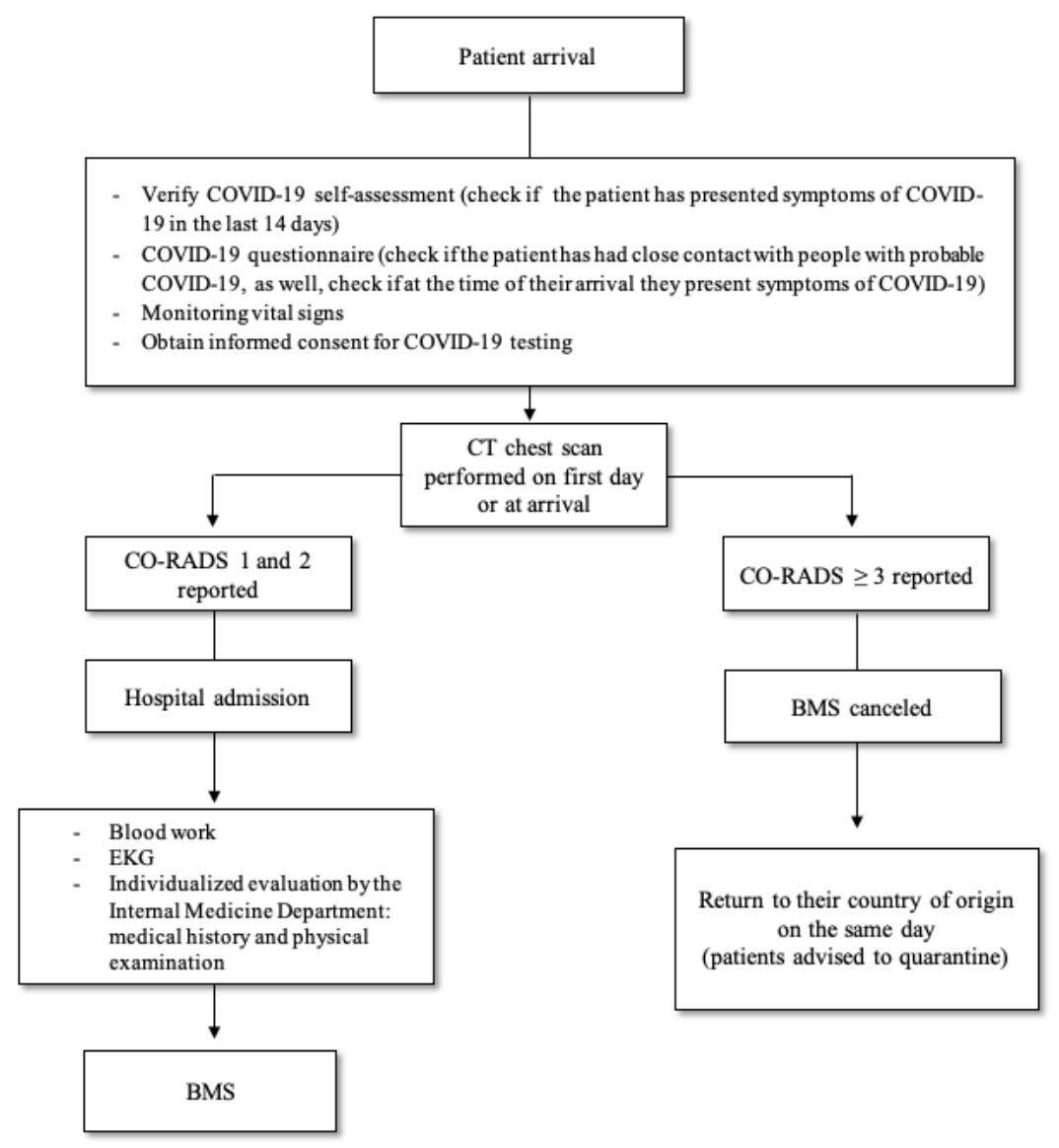

Figure 2

Preoperative selection algorithm COVID-19 = coronavirus disease 2019; CT chest = computed tomography of the chest; BMS = bariatric and metabolic surgery; EKG = electrocardiogram.

\section{Supplementary Files}

This is a list of supplementary files associated with this preprint. Click to download.

- Appendix.docx 